\title{
Uso de prótese e autopercepção de saúde bucal entre idosos
}

\author{
Marina Giovanna Alves Ribeiro ${ }^{1}$; Larissa Ledo Pereira Sant'Ana ${ }^{2}$; Luciana Thaís Rangel Souza ${ }^{3}$; \\ Jônatas Pereira do Prado ${ }^{4}$
}

\begin{abstract}
Resumo: No âmbito da Odontologia, a condição de saúde bucal dos idosos tem revelado uma prática assistencial mutiladora, acarretando múltiplas exodontias e reabilitações protéticas totais. O uso da prótese total removível afeta os idosos nos aspectos emocionais e sociais, visto que a adaptação a prótese, carece da dedicação emocional e funcional. Neste contexto, o objetivo desta pesquisa foi analisar o nível de autopercepção de saúde bucal de 30 idosos de ambos os sexos que fazem uso de prótese total removível e os efeitos na qualidade de vida. Para execução da pesquisa utilizou-se o questionário Geriatric Orak Health Assessment Index), a autopercepção será classificada pela soma das respostas em ótima (34 a 36) pontos, regular (31 a 33) e ruim $(\leq 30)$. O escore média do foi considerado baixo 26,42 que correspondeu 76,67\% nos voluntários avaliados. Os resultados indicam que os idosos mesmo apresentando autopercepção baixa classificam a saúde bucal como satisfatória.
\end{abstract}

Descritores: Idosos; Autoimagem; Prótese Total; Qualidade de Vida.

\section{Use of prosthesis and self-perception of oral health among the elderly}

\begin{abstract}
In the area of dentistry, the oral health condition of the elderly has revealed a mutilating care practice, leading to multiple exodontia and total prosthetic rehabilitation. The use of total removable prosthesis affects the elderly in the emotional and social aspects, since adaptation to the prosthesis lacks the emotional and functional dedication. In this context, the objective of this research was to analyze the level of self-perception of oral health of 30 elderly people of both sexes who make use of total removable prosthesis and the effects on quality of life. The self-perception will be classified by the sum of the responses in optimal (34 to 36) points, regular (31 to 33 ) and poor $(\leq 30)$. The mean score was considered low, 26,42 which corresponded to $76.67 \%$ in the volunteers evaluated. The results indicate that the elderly, even with low self-perception, classify oral health as satisfactory.
\end{abstract}

Keywords: Elderly; Self image; Total prosthesis; Quality of life.

\footnotetext{
${ }^{1}$ Graduanda do curso de Odontologia da Faculdade Independente do Nordeste. Vitória da Conquista - BA - Brasil. Contato: marinagiovannalves@ hotmail.com;

${ }^{2}$ Professora do curso de Odontologia da Faculdade Independente do Nordeste. Vitória da Conquista - BA - Brasil. Contato: larissaledo@hormail.com;

${ }^{3}$ Graduanda do curso de Odontologia da Faculdade Independente do Nordeste. Vitória da Conquista - BA - Brasil. Contato: luthaisrs@gmail.com;

${ }^{4}$ Graduando do curso de Odontologia da Faculdade Independente do Nordeste. Vitória da Conquista - BA - Brasil. Cotato: prado.jonatas@gmail.com.
} 


\section{Introdução}

Com a transição demográfica, proveniente do envelhecimento da população, bem como da redução da taxa de mortalidade e fecundidade, estima-se que no ano de 2020, o número de idosos no Brasil alcançará 32 milhões (AZEVEDO, et al. 2017; ROSEDO, et al., 2017).

Diante do processo de envelhecimento foram necessárias remodelações nas políticas públicas de atenção ao idoso devido a precariedade que se encontrava o mesmo. Estas melhorias teriam como propósito atender as necessidades desse grupo, já que as enfermidades como as doenças crônicas, degenerativas e infectocontagiosas, tem afetado em maior abundância a sociedade (AGOSTINHO; CAMPOS; SILVEIRA; EDENTULISMO, 2015; CARNEIRO, et al., 2013; SOUZA, et al., 2016).

Dados de levantamento epidemiológico de saúde bucal brasileiro de 2010, consideram que metade da população idosa se apresenta edêntula (57,7\%). Reflexo da prática assistencial mutiladora ${ }_{2}$ ocasionando um número exacerbado de extrações, e reabilitação protética dentária total como método de eleição para essa circunstância (SILVA; DEMARCO; FELDENS, 2015). A perda dentária transparece o cuidado com a saúde bucal, fatores socioeconômicos e do nível de escolaridade em saúde do indivíduo. O edentulismo é declarado como problema de saúde pública e exibe influências estéticas, psicológicas, sociais, mastigatórias, deglutição, bem como na fala e na gustação (AGOSTINHO; CAMPOS; SILVEIRA; EDENTULISMO, 2015; KREVE; ANZOLIN , 2016; LEWANDOWSKI; BÓS, 2014).

Os pacientes que fazem o uso de prótese total removível podem expressar insatisfação em relação aos aspectos emocionais e sociais ${ }_{-}^{-}$quando comparados com indivíduos com dentição natural. A adaptação a prótese removível requer o empenho funcional e emocional do paciente (YEN, et al., 2015). Essa insatisfação pode implicar na qualidade de vida, a qual remete ao bem-estar psicológico, físico e social do mesmo. Apesar disso ${ }_{2}$ a satisfação do idoso com a prótese demanda a avaliação para averiguar se a reabilitação oral está, em um âmbito mais amplo $_{2}$ satisfazendo as necessidades nos aspectos funcionais, biológicos, psicológicos e sociais do indivíduo (VERONEZ, et al., 2014).

Com a avaliação da autopercepção o profissional é capaz de identificar o impacto que a doença tem sobre o paciente, além de ser possível verificara gravidade das disfunções proveniente da situação de saúde. É de suma importância a autoavaliação nos tratamentos 
reabilitadores com prótese dentaria total. Deste $\operatorname{modo}_{2}$ a reabilitação pode ser por demanda pessoal, econômica e da autopercepção da necessidade de prótese total (SOUZA, et al., 2016). Faz-se necessário estimar a prevalência da autopercepção de idosos desdentados, para desta forma assinalar os fatores associados a autopercepção. Nesse sentido, o objetivo do presente estudo foi averiguar o nível de satisfação em relação à saúde bucal de indivíduos com 60 anos ou mais que fazem uso de prótese total e os impactos na QVRSB dos mesmos.

\section{Material e Métodos}

\section{Considerações Éticas}

O presente estudo foi submetido ao Comitê de Ética em Pesquisa segundo resolução vigente para Ética em Pesquisa em Seres Humanos n 466/12 do Conselho Nacional de Saúde, obtendo um parecer de aprovação, n² 2.653.029. Dentre os critérios de inclusão estão, ser idoso, com idade igual 60 ou maior, de ambos os sexos, sem distinção socioeconômica, concordar em participar do estudo, ausência de deficiência física e/ou cognitiva. A atuação do participante foi de forma voluntária com ausência de remuneração, realizou-se a explicação do objetivo e a justificativa do estudo. Esta pesquisa não obteve financiamento para a sua execução.

\section{Amostra}

O questionário GOHAI (Geriatric Orak Health Assessment Index) na versão portuguesa foi destinada a 30 idosos, os quais frequentam o Abrigo Nosso Lar, a Clínica Odontológica da Faculdade Independente do Nordeste e um consultório particular. Ambos situados na cidade de Vitória da Conquista - BA. Cautelas foram adotadas para evitar influência nas respostas do entrevistado, dentre elas estão: a entrevista feita face-a-face, individuais e em uma sala reservada. 


\section{Instrumento de recolha de dados}

O Índice GOHAI (Geriatric Orak Health Assessment Index) avalia a saúde bucal sobre a qualidade de vida dos idosos através da autopercepção.Consiste em utilizar o método de questionário dirigido, contendo 12 perguntas associadas à fala,à alimentação e à deglutição (função física); dor ou desconforto e uso de medicamentos para alivio do mesmo; satisfação e insatisfação com a saúde bucal e privação do contato social por problemas orais (função psicossocial).

De acordo com os Atchison e Dolan ${ }^{2}$ (1990) as respostas podem obter de 3-6 categorias. O presente estudo utilizou-se escala de frequência simples com 3 categorias, distribuindo os pesos de 1 a 3, assim sendo:(1) sempre, (2) às vezes e (3) nunca, no entanto as questões 3,5 e 7 que apresentam seus valores invertidos. Deste modo, o escore de cada colaborador pode oscilar de 12 a 36 pontos, quanto maior o escore melhor a autopercepção de saúde bucal e qualidade de vida. A partir da média de cada participante, foi feita a classificação da autopercepção de saúde bucal em,elevada (34 a 36$)$ pontos, moderada (31 a 33$)$ pontos e baixa $(\leq 30)$ pontos seguindo os autores.

\section{Análise de dados}

Os resultados obtidos foram tabulados e analisados descritivamente qualificando assim a autopercepção de saúde bucal de idosos que fazem o uso de próteses totais, utilizando software SPSS Statistical Package for the Social Sciences Statistics Base 22.0.

\section{Resultados}

Foram examinados 30 idosos, com média de idade de 71,6 anos, constituídos principalmente pelo sexo feminino (Tabela 1). A faixa etária que apresentou maior número de entrevistados foi 60-74 anos (n- 20) que corresponde a 66,67\% dos voluntários. 
Tabela 01. Classificação da amostra de acordo com o GOHAI

\begin{tabular}{ccccccccccc}
\hline Sexo & \multicolumn{2}{c}{ Ruim } & \multicolumn{2}{c}{ Regular } & \multicolumn{2}{c}{ Boa } & \multicolumn{2}{c}{ Ótimo } & \multicolumn{2}{c}{ Não sabe } \\
& $\mathrm{n}$ & $\%$ & $\mathrm{n}$ & $\%$ & $\mathrm{n}$ & $\%$ & $\mathrm{n}$ & $\%$ & $\mathrm{n}$ & $\%$ \\
\cline { 2 - 12 } Feminino & 1 & 3,33 & 3 & 10,00 & 13 & 43,33 & 3 & 10,00 & 1 & 3,33 \\
Masculino & 0 & 0 & 6 & 20,00 & 3 & 10,00 & 0 & 0 & 0 & 0 \\
\hline
\end{tabular}

$\mathrm{Na}$ análise da autopercepção de saúde bucal obteve-se: 3,33\% ruim; 30\% regular; $53,33 \%$ boa; $10 \%$ ótimo e 3,33\% da amostra não soube classificar sua saúde bucal. O sexo feminino qualificou a avaliação de saúde bucal com maior percentual 43,33\% (n=13) como "boa", enquanto no sexo masculino $20 \%(n=6)$ classificaram a saúde bucal como "regular" (Tabela 2).

Tabela 02. Avaliação de saúde bucal do idoso: número e porcentagem

\begin{tabular}{lccc}
\hline Variável & Média & IC95\% & Desvio padrão \\
\hline GOHAI & 26,42 & $24,5-28,2$ & $\pm 4,8$ \\
Dimensão Física & 6,1 & $5,2-7,0$ & $\pm 2,4$ \\
Dimensão psicossocial & 10,5 & $8,9-11,7$ & $\pm 3,7$ \\
Dimensão dor ou desconforto & 9,8 & $8,9-10,7$ & $\pm 2,3$ \\
\hline
\end{tabular}

Durante a mensuração de saúde bucal geriátrica segundo GOHAI, a maior parte dos voluntários entrevistados $(76,66 \%)$ relatou não ter limitado o contato com outras pessoas devido ao uso de prótese total. Quanto ao uso de medicamentos para alívio da dor apenas $(36,66 \%)$ fez uso de fármacos para redução da dor. O desconforto devido ao uso de prótese total pode acarretar na redução da quantidade de alimento ingerido pelo idoso, os voluntários participantes da pesquisa $(63,33 \%)$ afirmaram ter limitado o tipo ou a quantidade de alimento ingerido.

$\mathrm{Na}$ execução do índice GOHAI os escores médios foram inferiores aos limites superiores da escala de medidas do índice global (média=26,42) (Tabela 3), apontando que a autopercepção entre os idosos que fazem o uso de prótese total é considerada baixa. A maior 
parte dos voluntários $(76,67 \%)$ apresentaram baixa autopercepção de saúde bucal que correspondem a valores menores que 30; 16,67\% apontaram autopercepção moderada (resultados entre 31-33) e apenas 6,67\% autopercepção elevada (valores entre 34-36).

Tabela 03. Autopercepção da saúde bucal dos idosos: média, intervalo de confiança e desvio padrão do GOHAI.

As variáveis sexo, idade e autopercepção estabelecida pelo GOHAI constituiu maior percentual ( $n=16$ / 53,33\%) de autopercepção de saúde bucal baixa entre os idosos com idade entre 60-74 ano (Tabela 4). Os resultados analisados e relacionados as questões do GOHAI, foram segmentadas em três domínios: físico, dor e/ou desconforto e psicossocial.

\begin{tabular}{|c|c|c|c|c|c|c|c|c|}
\hline \multirow[t]{2}{*}{$\begin{array}{c}\text { Características } \\
\text { Sociodemográficas }\end{array}$} & \multirow[t]{2}{*}{$\mathrm{n}$} & \multirow[t]{2}{*}{$\%$} & \multicolumn{2}{|c|}{$\begin{array}{l}\text { Autopercepção de saúde } \\
\text { bucal baixa }(<30)\end{array}$} & \multicolumn{2}{|c|}{$\begin{array}{l}\text { Autopercepção de saúde } \\
\text { bucal moderada (31-33) }\end{array}$} & \multicolumn{2}{|c|}{$\begin{array}{c}\text { Autopercepção de saúde } \\
\text { bucal elevada (34-36) }\end{array}$} \\
\hline & & & $\mathrm{n}$ & $\%$ & $\mathrm{n}$ & $\%$ & $\mathrm{n}$ & $\%$ \\
\hline \multicolumn{9}{|l|}{ Sexo } \\
\hline Masculino & 9 & 30 & 8 & 26,67 & 1 & 3,33 & 0 & 0 \\
\hline Feminino & 21 & 70 & 15 & 50 & 4 & 13,33 & 2 & 6,67 \\
\hline \multicolumn{9}{|l|}{ Faixa etária (anos) } \\
\hline $60-74$ & 20 & 66,67 & 16 & 53,33 & 3 & 10,00 & 1 & 3,33 \\
\hline $75-84$ & 8 & 26,67 & 6 & 20,00 & 1 & 3,33 & 1 & 3,33 \\
\hline$\geq 85$ & 2 & 6,67 & 1 & 3,33 & 1 & 3,33 & 0 & 0 \\
\hline
\end{tabular}

Tabela 04. Classificação absoluta e percentual da amostra conforme sexo, idade e de autopercepção de saúde bucal estabelecida pelo GOHAI.

\section{Discussão}

No âmbito cientificoazem que se respalda ${ }_{2}$ a Odontologia apresenta uma carência a respeito da satisfação do paciente com relação ao tratamento. Desta forma ${ }_{2}$ tem se lançado mão do uso de meios para mensurar e conhecer a concepção do indivíduo em relação à saúde bucal, permitindo constatar falhas, ausência de informações, a concreta necessidade do tratamento, 
além de ponderar as prerrogativas estabelecidas pelo tratamento protético (BELONI; VALE; TAKAHASHI, 2013).

Diversos métodos de avaliação então sendo utilizados nos últimos 10 anos, tendo como objetivo de evidenciar a qualidade de vida relacionada a saúde bucal(QVRSB), fundamentado em cômputos dos aspectos físicos, psicológicos e sociais. Dentre os métodos ${ }_{2}$ temos o Índice Geriátrico de Avaliação de Saúde Bucal (GOHAI) e o perfil do impacto na saúde bucal (OHIP) (SCHUSTER, et al., 2017; SILVA; BONINI; BRINGEL, 2015).

No ano de 1990 Atchison e Dolan elaboraram o método Geriatric Oral Health Assessment Index (GOHAI), no qual evidencia o impacto da saúde bucal na rotina diária do idoso, de modo que ele compreenda o bem-estar, refletindo nos cuidados de saúde bucal (PALMA, et al., 2015; ROSENDO, et al., 2017).

Os idosos voluntários, com idade igual ou superior a 60 anos, denota uma autopercepção de saúde bucal baixa em mais da metade dos indivíduos, constituída assim principalmente por mulheres como mostra alguns estudos (NOGUEIRA. et al., 2017; RIHS, et al., 2012; SÔNEGO, et al., 2013). Quando comparado os valores exibido na pesquisa realizada na Faculdade de Odontologia de Piracicaba onde a população deste estudo apresentou percentual médio também inferior, denotasse que a amostra do estudo era apenas do sexo feminino (SILVA; SOUSA, 2006). Sendo assim tais valores explicados pelos censos 2011, onde evidencia que a hegemonia da população feminina é elevada à proporção que a idade amplifica (CARVALHO, et al,. 2016).

Os resultados obtidos, neste trabalho, pelo índice GOHAI atingiu uma média de 26,42, caracterizando uma auto-percepção de saúde bucal extremamente ruim dos idosos apreciados, inferior ao limite médio de auto percepção que corresponde uma pontuação superior a 30 . Pesquisas realizadas nas cidades de Araraquara, Campinas, Piracicaba e Rio Claro, São Paulo,, Silva; Souza et al. (2005), Silva; Held et al. (2011), Silva; Souza et al. (2006), Sônego; Porfírio et al. (2013), apontando escore de,27,7;33,9; 29,6; 33,2, exibiram uma mutabilidade na autopercepção em média e ruim entre os idosos de um mesmo estado.

O agente decisivo da autopercepção baixa foi a dimensão física, onde o padrão de mastigação, fala e deglutição é declarado pelos participantes como incômodo. No estudo realizado por Lima et al.(2013) o fator decisório que resultou na escore como ruim foi a função 
protética defeituosa, assim como fonética e estética, porem aqueles que foram entrevistados relataram que sua saúde bucal não carecia de melhorias.

Grande parte dos voluntários avaliou a condição de saúde bucal como satisfatória apenas 10 dos voluntários declararam como ruim ou regular, no entanto quanto computado os resultados do índice GOHAI revela condições deficientes, corroborando com estes dados os estudos de Silva et al. (2011) e Vasconcelos et al. (2012),contataram que idosos avaliam a saúde bucal como favorável mesmo não obtendo condições adequadas.

O sucesso da prótese total em idoso é concordante com a literatura, onde mostra, que a satisfação do paciente que faz o uso da prótese esta intimamente relacionada com a qualidade e funcionalidade que a prótese total desempenha (NASCIMENTO; ANDRADE; CÉSAR, 2015). Para os autores Nunes; Abegg (2008), a percepção de saúde bucal do idoso e compreendida apenas quando relata, sintomas como dor e sinais, ou dificuldade durante a mastigação, o que acaba afetando a aparência.

Acredita-se que a percepção dos idosos podem ser prejudicadas pelas convicções, bem como o valor conferido à perda da saúde do idoso afetado (HIRAMATSU; FRANCO; TOMITA, 2006). A qualidade de vida esta intimamente relacionada à saúde bucal, isso pode ser visível em especial nos idosos que apresentam alguma fragilidade biológica, criado obstáculos no acesso a tratamentos odontológicos, sobretudo postergando a visita ao CirurgiãoDentista em consequência das demandas de saúde. Em contrapartida, a condição de saúde bucal prejudicada pode colaborar no desenvolvimento de distúrbios alimentares, com isso uma deficiência nutricional (MESAS, et al., 2010) . A condição de saúde bucal insatisfatória desencadeia infecções e inflamações, favorecendo o risco de fragilidade. Com isso idosos vulneráveis apresentam complexos problemas bucais, interferindo no comprometimento funcional, induzindo a avaliação de saúde bucal de maneira negativa (SUMI, et al., 2009).

O presente trabalho teve como limitação os locais de aplicação do instrumento de avaliação, devido a frequência reduzida de pacientes que se enquadrava no estudo, bem como mais conveniente para submissão ao CEP. Desta forma, se faz necessário que novas pesquisas sejam realizadas para comprovação e esclarecimento desta temática, com tempo de pesquisa mais amplo e amostra superior. 


\section{Conclusão}

De acordo com o índice GOHAI, os voluntários do estudo classificaram a autopercepção de saúde bucal como "baixa. No entanto na autoavaliação o sexo feminino considerou a saúde bucal como boa, isso demonstra que as idosas mesmo apresentando condições inadequadas de saúde bucal declaram como favorável.

Com base nos dados obtidos é de suma importância a relação entre saúde geral e saúde bucal, onde é capaz de fornecer dados para assegurar a implementação de programas de saúde bucal para a promoção do bem-estar e o autocuidado na terceira idade.

\section{Referências}

AGOSTINHO ACMG, CAMPOS ML, SILVEIRA JLGC. Edentulismo, uso de prótese e autopercepção de saúde bucal entre idosos. Rev. odontol. UNESP, v.44, n.2, p.74-9 2015 . Disponível em: http://www.scielo.br/scielo.php?script=sci_arttext\&pid=S1807-257720 $150002000 \quad 74 \& \operatorname{lng}=\mathrm{en}$. Acesso em: 28/02/2018

ATCHISON KA, DOLAN TA. Development of the Geriatric Oral Health Assessment Index. J Dent Educ, v. 54, n.11, p.680-7, 1990. Disponível em: https://www.ncbi.nlm.nih.gov /pubmed/2229624

AZEVEDO JS. et al. Uso e necessidade de prótese dentária em idosos brasileiros segundo a Pesquisa Nacional de Saúde Bucal (SBBrasil 2010): prevalências e fatores associados. Cad. Saúde Pública, v.33, n.8, 2017. Disponível em: http://www.scielo.br/scielo.phpscript=sci_arttext\&pid $=$ S0102311X2017000805002\&lng=en. Acesso em: 02/02/2018

BELONI WB, VALE HF, TAKAHASHI JMFK. Avaliação do grau de satisfação e qualidade de vida dos portadores de prótese dental. RFO, v.18, n.2, p.160-4, 2013. Disponível em:http://seer.upf.br/index.php/rfo/article /view/3255/2492. Acesso em: 28/02/2018

CARNEIRO SDRM. et al. Prevalência de Edentulismo e situação periodontal em idosos atendidos no curso de Odontologia da Universidade de Fortaleza (UNIFOR). Braz J Periodontol. Sociedade brasileira de Periodontologia, v.23, n.2, p.07-10, 2013. Disponível em: http://www.revista sobrape.com.br/arquivos/2013/junho/REVPERIO_ JUN_2013_PUBL_SITE_PAG-07_A_10.pdf. Acesso em: 28/02/2018.

CARVALHO C. et al. Self-perception of oral health in older adults from an urban population in Lisbon, Portugal. Rev. Saúde Pública, v.50, n.13, p.53, 2016. Disponível em: http://www.scielo.br/scielo.php?script=sci_arttext\&pid=S003489102016000100235\&lng=en. 
HIRAMATSU DA, FRANCO LJ, TOMITA NE. Influência da aculturação na autopercepção dos idosos quanto à saúde bucal em uma população de origem japonesa. Cad. Saúde Pública. v.22, n.11, p.24412448, 2006. Disponível em: http://www.scielo.br/scielo. php?script=sci_arttext\&pid=S0102$311 X 2006001100018 \& \operatorname{lng}=$ en.

KREVE, S., ANZOLIN D. Impacto da saúde bucal na qualidade de vida do idoso. Rev. Kairós Gerontologia, v.19, n.22, p.45-59, 2016. Disponível em: http://www.scielo.br/pdf/csc/ v16n7 /31.pdf. Acesso em: 28/02/2018

LEWANDOWSKI A, BÓS ÂJG. Estado de saúde bucal e necessidade de prótese dentária em idosos longevosStateof oral healthandnecessityofimplantdentistry in theoldestold. Rev. Assoc. Paul. Cir. Dent. [serial onthe Internet], v.68, n.2, p.155-8, 2014. Disponível em: http://revodonto.bvsalud.org/scielo.php?script=sci_arttext\&pid=S0004-5276201400 0200 014\&lng=en. Acesso em: 03/02/2018

LIMA JM. et al. Avaliação da percepção da saúde bucal e qualidade de vida de idosos do Projeto de Extensão da Universidade Católica de Brasília (UCB) - Centro de Convivência de Idosos (CCI) a partir da utilização do instrumento GOHAI. Rev. Portal de Divulgação, v.36, Ano IV, 2013. Disponível em: https://www.jornaldosite.com.br/arquivo bucalGOHAIQualidade\%20de\%20vida\%20.pdf

/Odontogeriatria/31condicao

MESAS AE. et al. Saúde bucal e déficit nutricional em idosos não institucionalizados em Londrina, Paraná, Brasil. Rev. bras. Epidemiol, v.13,n.3, p.434-445, 2010. Disponível em: http://www.scielo.br/scielo.php?script=sci_arttext\&pid=S1415-790X2010000300007 \&lng=en.

NASCIMENTO AR, ANDRADE FB, CÉSAR CC. Validade e utilidade da autopercepção de necessidade de tratamento odontológico por adultos e idosos. Cad. Saúde Pública, v.31, n.8, p.17651774, 2015. Disponível em: http://www.scielo.br/scielo.php ?script=sci_ arttext\&pid=S0102311X2015000801765\&lng=en.

NOGUEIRA CMR. et al. Self-perceived oral health among the elderly: a household-based study. Rev. bras. geriatr. Gerontol, v.20, n.1, p.7-19, 2017. Disponível em: http://www.scielo.br/scielo.php?script=sci_arttext\&pid=S180998232017000100007\&lng=en.

NUNES CI, ABEGG C. Factors associated with oral health perception in older Brazilians. Gerodontology, v.25, n.1, p.42-8, 2008. Disponível em: https://onlinelibrary.wiley.com/ doi/abs/10.1111/j.1741-2358.2007.00163.x

PALMA JM. et al. Edentulismo e autopercepção em saúde bucal em idosos de um município do nordeste brasileiro.Rev Pesq Saúde, v.16, n.3, p.144-8. . 2015 Disponível em: http://www.periodicos eletronicos.ufma.br/index.php/ revistahuufma/article/view/4510. Acesso em: 28/02/2018

RIHS LB. et al. Autopercepção em saúde bucal em idosos frágeis. Rev assoc paul ciR dent, v.66, n.2, p. 105-9, 2012. Disponível em:http://revodonto.bvsalud.org/pdf/aped/ v66n2 /a04v66n2.pdf

ROSENDO RA. et al. Autopercepção de saúde bucal e seu impacto na qualidade de vida em idosos: uma revisão de literatura. RSC, v.6, n.1, p.89-102, 2017. Disponível em:http://www.ufcg.edu.br/revistasaudeeciencia/index.php/RSCUFCG/article/view/307.Acesso em: 03/02/2018. 
SCHUSTER AJ. et al . Short-termqualityoflifechangeperceivedbypatientsaftertransitionto mandibular overdentures. Braz. oral res, v.31, n.5, p.01-09, 2017. Disponível em: http://www.scielo.br/scielo.php?script=sci_arttext\&pid=S180683242017000100220\&lng $=$ en. Acesso em: 14/02/2018.

SILVA AE, DEMARCO FF, FELDENS CA. Qualidade de vida relacionada à saúde bucal e fatores associados em idosos do sul do Brasil. Gerodontologia, v.32, n.1, p. 35-45. 2015. Disponível em: https://onlinelibrary.wiley.com/doi/abs/10.1111/ger.12050.

SILVA BLA, BONINI JA, BRINGEL FA. Condição de saúde bucal de idosos institucionalizados em Araguaína/TO. Braz J Periodontol. Sociedade brasileira de Periodontologia, v.25, n.1, p.07-13, 2015. Disponível em: http://docplayer.com.br /10933273-Condicao-de-saude-bucaldeidososinstitucionalizados-em-araguaina-to-oral-health-status-of-institucionalized-elderly-inaraguaina to.html. Acesso em:28/02/2018.

SILVA DD et al. Autopercepção da saúde bucal em idosos e fatores associados em Campinas, SP, 20082009. Rev. Saúde Pública, v.45, n.6, p.1145-11532011. Disponível em: http://www.scielo.br/scielo.php?script=sci_arttext\&pid=S0034-89102011000 600017 \&lng=PT.

SILVA DD, SOUSA MLR, WADA RS. Autopercepção e condições de saúde bucal em uma população de idosos. Cad. Saúde Pública, v.21, n. 4, p. 1251-1259, 2005 Disponível em: http://www.scielo.br/scielo.php?script=sci_arttext\&pid=S0102-311X200500040002 $8 \& \operatorname{lng}=$ en. http://dx.doi.org/10.1590/S0102-311X2005000400028.

SILVA EFA, SOUSA MLR. Autopercepção da saúde bucal e satisfação com a vida em mulheres idosas usuárias de prótese total. Revista de Odontologia da Universidade Cidade de São Paulo, v.18, n.1, p.61-65, 2006. Disponível em: http://arquivos.cruzeiro dosuleducacional.edu.br/principal/old/revista_odontologia/pdf/1_janeiro_abril_2006/autopercepcao_sa ude_bucal.pdf

SÔNEGO PI. et al. Autopercepção de Saúde Bucal de Idosos Institucionalizados e não Institucionalizados do Município de Araraquara- SP. Revista Brasileira Multidisciplinar, v.16, n.2, p. 37-53, 2013. Disponível em: http://revistarebram.com/index .php/revistauniara /article/view/62/41.

SOUZA JGS. et al. Autopercepção da necessidade de prótese dentária total entre idosos brasileiros desdentados. Ciênc. saúde coletiva, v.21, n.11, p.3407-14, 2016. Disponível em:http://www.scielo.br/scielo.php?script=sci_arttext\&pid=S141381232016001103407\&lng=en. Aces so em: $12 / 02 / 2018$.

SUMI Y. et al. Relationship between oral function and general condition among Japanese nursing home residents. Arch Gerontol Geriatr. v.48, n.1, p.100-5, 2009. Disponível em: https://www.ncbi.nlm.nih.gov/pubmed/18096255

VASCONCELOS LCA. et al. Autopercepção da saúde bucal de idosos de um município de médio porte do Nordeste brasileiro. Cad. Saúde Pública, v.28, n.6, p. 1101-1110, 2012. Disponível em: http://www.scielo.br/scielo.php?script=sci_arttext\&pid=S0102-311X201 2000600009\&lng=en.

VERONEZ FC. et al. User satisfaction with complete dentures made by the public network of a city in southern Santa Catarina. RGO, Rev. Gaúch. Odontol, v. 62, n.2, p. 123-127. 2014. Disponível em: 
http://www.scielo.br /scielo.phpscript=sci_arttext\&pid=S198 186372 014000200123\&lng=en. Acesso em: 06/02/2018

YEN YY. et al. Impact of removable dentures on oral health-related quality of life among elderly adults in Taiwan. BMC Oral Health, v. 15, n.1, 2015. Disponível em: https://bmcoralhealth. biomedcentral.com/articles/10.1186/1472-6831-15-1\#Sec8. Acesso em: 28/02/2018

\section{Como citar este artigo (Formato ABNT):}

RIBEIRO, Marina Giovanna Alves; SANT'ANA, Larissa Ledo Pereira; SOUZA, Luciana Thaís Rangel; PRADO, Jônatas Pereira do. Uso de prótese e autopercepção de saúde bucal entre idosos. Id on Line Rev.Mult. Psic., 2018, vol.12, n.42, p. 1203-1214. ISSN: 1981-1179.

Recebido: 19/10/2018;

Aceito: 23/10/2018 